\title{
Printing polymer optical Waveguides on conditioned transparent flexible Foils by using the Aerosol Jet Technology
}

\author{
Thomas Reitberger*a, Gerd-Albert Hoffmann ${ }^{\mathrm{b}}$, Tim Wolfer ${ }^{\mathrm{b}}$, Ludger Overmeyer ${ }^{\mathrm{b}}$, Joerg Franke \\ ${ }^{a}$ Institute for Factory Automation and Production Systems, Friedrich-Alexander-University \\ Erlangen-Nuremberg, Fuerther Strasse 246b, 90429 Nuremberg, Bavaria, Germany. \\ ${ }^{b}$ Institute of Transport and Automation Technology, Leibniz Universitaet Hannover, \\ An der Universitaet 2, 30823 Garbsen, Lower Saxony, Germany.
}

\begin{abstract}
The optical data transfer is considered as the future of signal transfer due to its various advantages compared to conventional copper-based technologies. The Aerosol Jet Printing (AJP) technology offers the opportunity to print materials with high viscosities, such as liquid transparent polymer adhesives (epoxy resins), on almost any possible substrate material and even in third dimension. This paper introduces a new flexible and comparatively cost-effective way of generating polymer optical waveguides through AJP.

Furthermore, the conditioning of the substrate material and the printing process of planar waveguides are presented. In the first step, two lines with hydrophobic behavior are applied on foil material (PMMA, PVC, PI) by using a flexographic printing machine. These silicone based patterns containing functional polymer form barriers for the core material due to their low surface energy after curing. In the second step, the core material (liquid polymer, varnish) is printed between the barrier lines. Because of the hydrophobic behavior of the lines, the contact angle between the substrate surface and the liquid core material is increased which yields to higher aspect ratio. The distance between the barrier lines is at least $100 \mu \mathrm{m}$, which defines the width of the waveguide. The minimum height of the core shall be $50 \mu \mathrm{m}$. After UV-curing of the core polymer, the cladding material is printed on the top. This is also applied by using the AJP technology. Various tests were performed to achieve the optimal surface properties for adequate adhesion and machine process parameters.
\end{abstract}

Keywords: Flexographic printing, Aerosol Jet Printing, polymer, optical, waveguide, additive manufacturing

\section{INTRODUCTION}

The future of high-speed data and signal transfer will hardly be possible using copper-based conductive technologies, regarding the massively growing demands of faster availability and bigger pockets of data (see Figure 1). For long distances like transatlantic communication, optical glass fiber technology is already in use. Currently there is no other technology providing higher transfer rates at lower attenuation with higher cost effectiveness. For distances shorter than $100 \mathrm{~m}$, polymer optical fibers (POF) are continuously gaining significance as a promising alternative to copper cables and to copper circuit paths on board level. [1]

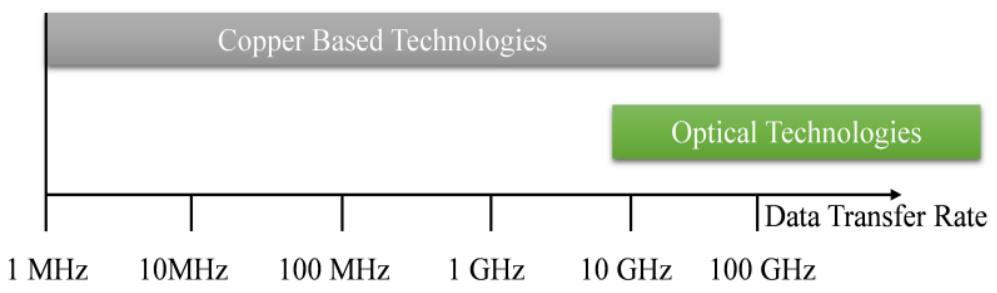

Figure 1. Potential data transfer rates of copper and optic technologies [1]

Presently, the board supply systems are prepared on a rigid bed of nails manually. The additive manufacturing is a key technology that offers a complete new perspective, when thinking of generating an innovative on-board supply system. There is a huge potential of saving weight for automotive, aircraft or space applications by directly printing the 
conductive paths on the components. Especially in aerospace parts, every bit of weight saving means an enormous impact on saving fuel, yielding to cost and environmental benefits.

This paper shows the latest results of a new and seminal way of producing polymer optical waveguides (POW) on polymethylmethacrylate (PMMA) foil, by using the combination of Aerosol Jet Printing and Flexographic Printing. Using PMMA-foil as lower cladding material saves at least one step of production. The main advantages of this process (OPTAVER-Process) are the short process chain, which is quiet similar to Inkjet Printing shown in Figure 2, compared to most of the common manufacturing technologies and the shape of the POWs, which is not quadrangular like most of today's additive generated waveguides.

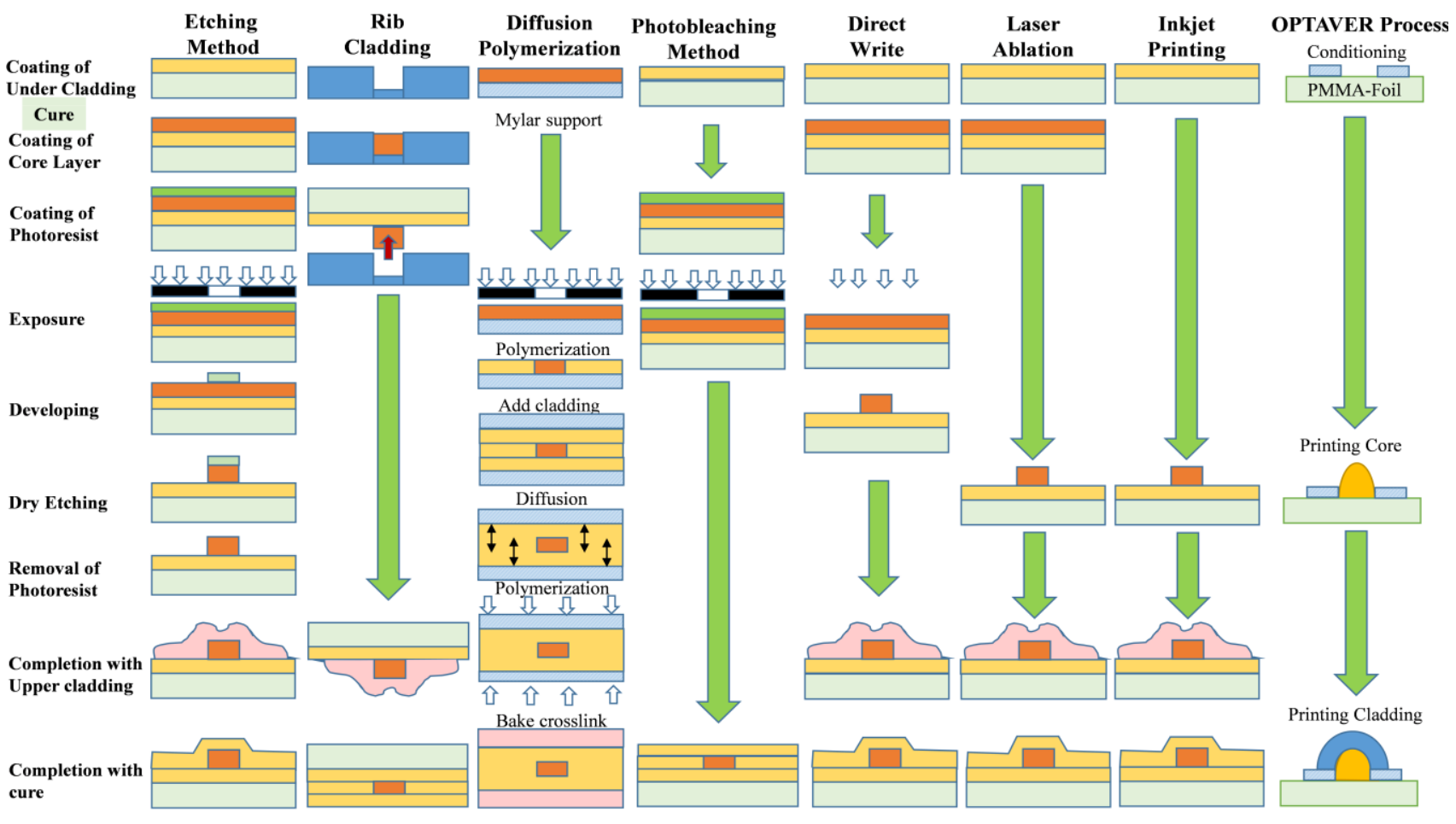

Figure 2. Overview of state-of-the-art production chains for polymer optical waveguides [1]

\section{OPTAVER-PROCESS}

\subsection{Flexographic-Printing}

Flexographic printing is positioned as a relief printing method where structures on the printing form are copied onto a substrate, which is a flexible PMMA foil in this case. The UV-varnish used for the printing process is transferred from the ink supply to the anilox roller. This cylinder has micrometer scaled cells on its surface and wets the printing form with ink.

The Optaver process uses a special structure to print a pair of lines out of functional polymer containing silicone. These lines have a certain distance and act like regional barriers for subsequent applied waveguide material with the AJP (Fig. 3). The conditioning line has a hydrophobic behavior, forcing the liquid on top to create waveguides by selfassembly and forms a parabolic cross section [2].

The distance between these conditioning lines can vary between 10 and $300 \mu \mathrm{m}$. Most important process parameters for Flexographic printing of high-resolution lines are the infeed of the printing form to the substrate material and the combination of chosen anilox cell size and stamp width. They manipulate the shape of the conditioning lines and the building of squeezing borders [3]. Because of the effect to the waveguide material its quality directly affects the deviation concerning the cross sectional width. 


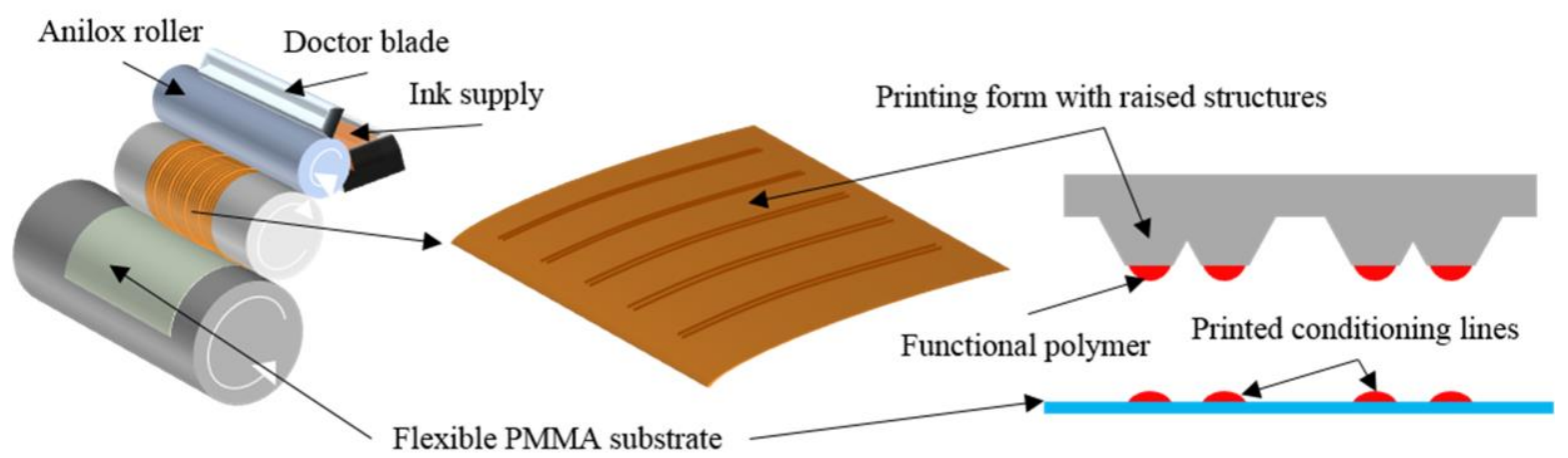

Figure 3. Flexographic printing mechanism and printed conditioning lines with adapted printing form

\subsection{Aerosol Jet Printing}

After conditioning the substrate material by Flexographic Printing, the next step is the Aerosol Jet Printing of the core material and curing it. In the last step, the cladding is applied on the top and UV-cured (see Fig. 2).

The Aerosol Jet process itself is shown in Figure 4. The basic principle of the pneumatic aerosol generation (5) is the nebulization of a liquid ink material (6) (in most cases silver ink; here: different kinds of UV-curing varnishes) by using an atomizer gas (1). The generated fine mist is transferred into the virtual impactor (3) where a second gas stream the socalled exhaust gas (2) withdraws the finer droplets by suction. The bigger ones fall down because of gravity and so a mostly homogeneous mist is formed. After that, the sheath gas stream (4) focuses the droplets while they pass the nozzle (8) and hit the substrate material. As the aerosol is generated continuously once the gas streams are started, there is a shutter (7), which is formed like a little spoon, to provide the ability of interrupting the ongoing Aerosol Jet stream to print a complex layout. There need to be a small gap between the nozzle and the shutter to avoid blockage of the nozzle with accumulated ink. [4]

The process is highly suitable to print on three-dimensional surfaces, as the distance between the nozzle and the substrate is flexible $(<10 \mathrm{~mm})$. Another main advantage is the fine pitch between the printed lines which is about $20 \mu \mathrm{m}$. [4]

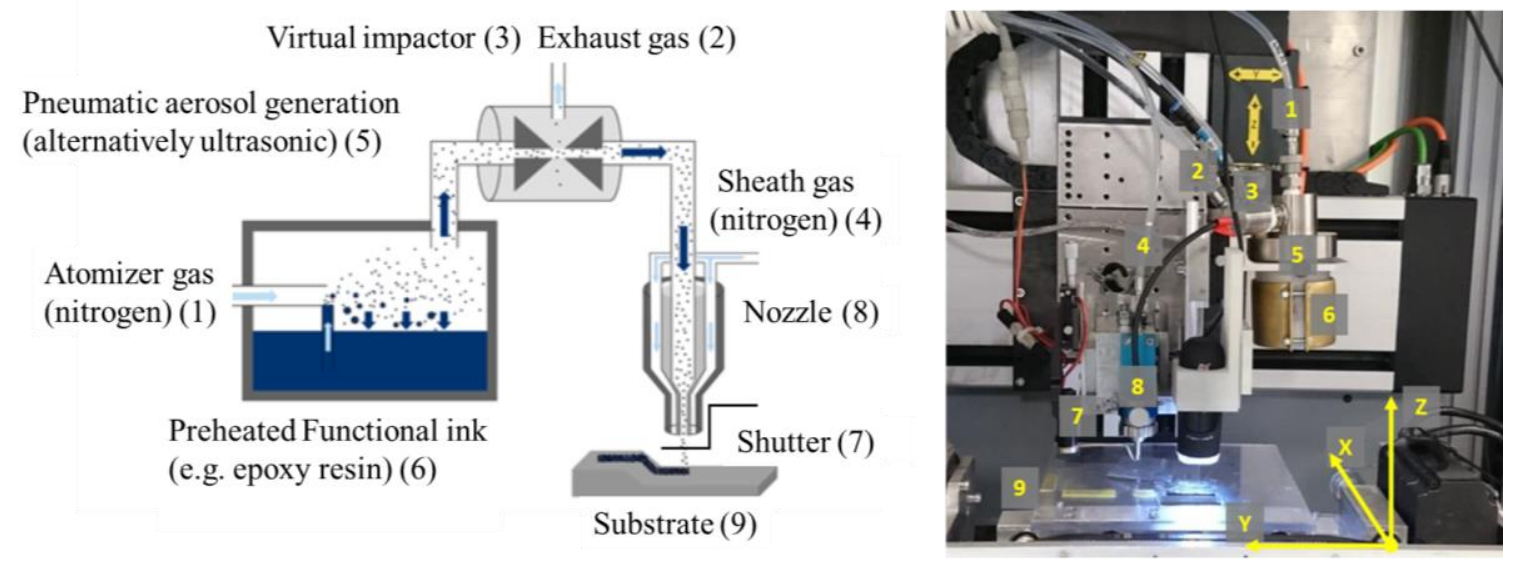

Figure 4. Left: schematic functional principle of the Aerosol Jet system (pneumatic atomization) [4]; Right: Physical laboratory setup

\section{GENERAL REQUIREMENTS AND SYSTEM ADJUSTMENTS}

\subsection{Influences of different surface treatments to the printing image on the substrate material}

As preliminary results for printing of POWs showed that the chosen surface treatment had an influence on the outcome of the printed lines, different ways of surface treatment were tested on PMMA, polyvinyl chloride (PVC) and polyimide (PI) foils. [5] PVC and PI foils were tested to get reference data for the PMMA foil used for the OPTAVER-process. Results of the tested varnishes (OrmoCore and Jaenecke \& Schneemann 390119) can be seen in Fig. 5. 
The foils (1-3\&10-12; blue) are PVC, the foils (4-6\&13-15 grey) PMMA and the remaining orange ones (7-9\&16-18) are PI respectively. On the left hand side, the ink used was OrmoCore on the right hand side it was J+S. From left to right in each row beginning with untreated, then isopropanol and finally plasma treated substrate material respectively.

OrmoCore
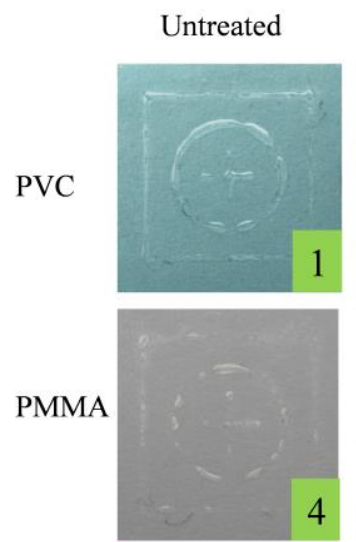

PI

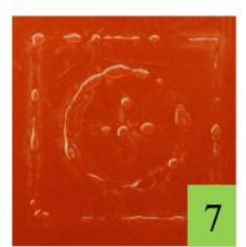

Isopropanol
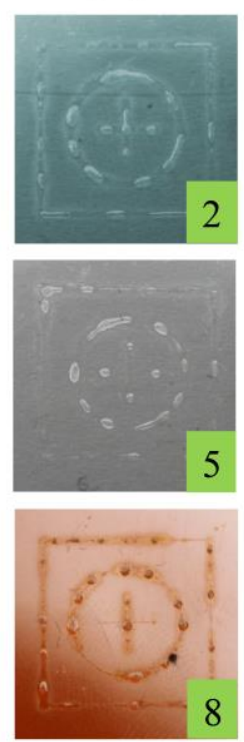

Plasma
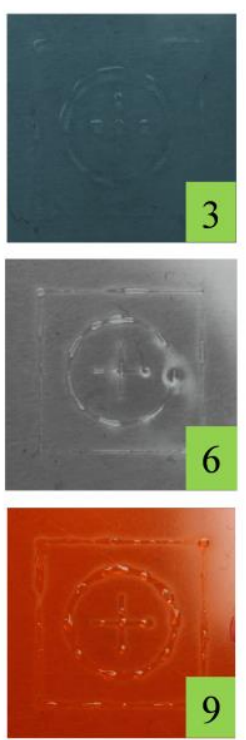

$\mathbf{J}+\mathbf{S}$
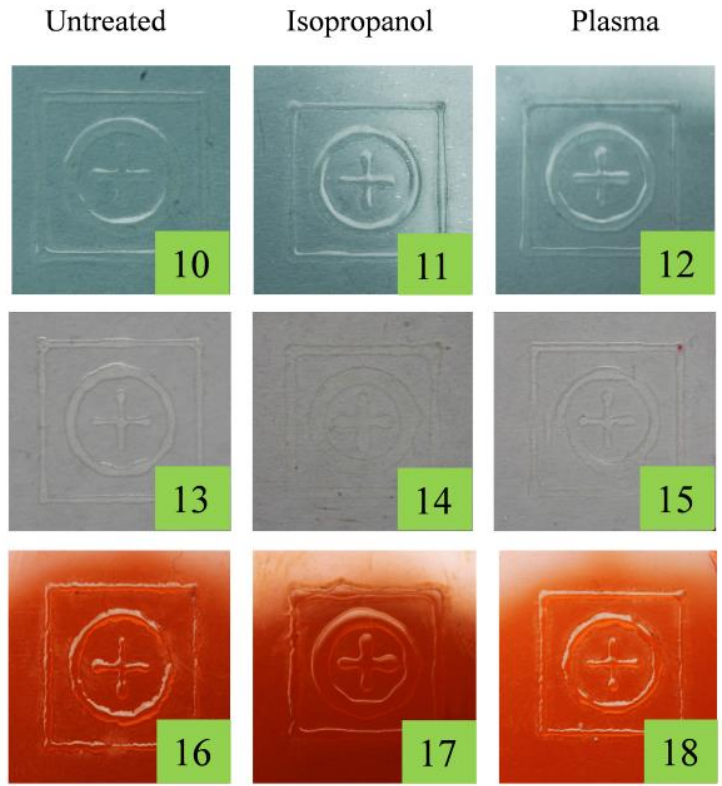
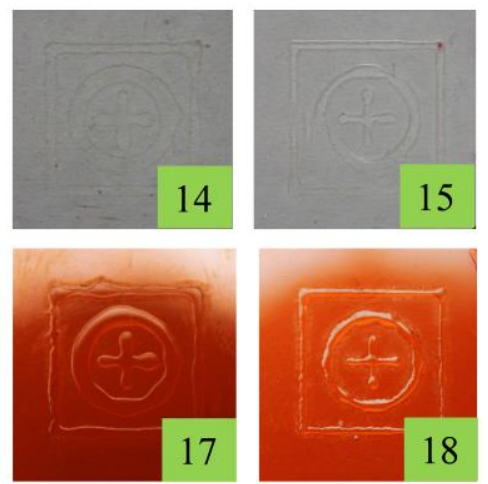

Figure 5. Different surface treatments and their influence on the print image. Left: OrmoCore on PVC, PMMA and PI substrate; Right: Clear varnish Jaenecke \& Schneemann 390119 on PVC, PMMA and PI substrate

Despite the results in the past, the current tests show that neither of the chosen surface treatments have strong influence on the printed structure. Regarding OrmoCore the best results were made on PVC and untreated substrate (1). The use of isopropanol leads to a higher droplet building $(2,5$, and 8$)$. Air plasma showed similar printing results as the untreated material. Overall, the OrmoCore material does not meet the requirements of the necessary print image (mostly droplets, no homogeneous lines).

$\mathrm{J}+\mathrm{S}$ indicated much better printing structures. Here straight edges and continuous lines can be seen. The best results could be achieved using PVC and PMMA as substrate material $(11,12$, and 15). On PI, straight edges could not be printed continuously. The usage of isopropanol led to broader lines while almost no significant effect was observed by plasma treatment.

These results depict that, for the here used inks, no pretreatment of the substrate material is proposed. For cleaning purposes, air plasma should be used instead of isopropanol.

\subsection{Machine parameters}

Based on results of previous investigations, see [6], [7], various tests were performed for developing the the machine parameters for the polymer inks. Especially for J+S, the following settings turned out to work best:

The distance between the nozzle and the substrate should not exceed $10 \mathrm{~mm}$ otherwise the overspray to the left and the right side of the printed line is growing massively. A sheath gas setting of $60 \mathrm{sccm}$ turned out to be the best choice for the used $300 \mu \mathrm{m}$ ceramic nozzle. Another important factor is the difference between the atomizer and the exhaust gas, which also showed the best results while using $60 \mathrm{sccm}$. The preheating temperature of the ink leads to a higher viscosity. The minimum temperature to achieve continuously proper results is $45^{\circ} \mathrm{C}$ and can be turned up until $60{ }^{\circ} \mathrm{C}$. The quality (no droplets or defects inside the printed structure) of the waveguides is higher using feed rates up to $3000 \mathrm{~mm} / \mathrm{min}$ and multiple numbers of layers $(10<\mathrm{X}<400$ depending on the feed rate) instead of printing one complete line in one cycle at a lower speed. 


\subsection{Mass flow measurement for reaching the maximum amount of ink between the barrier lines}

Mass flow measurements were done with different polymer inks, to know the throughput of varnish per minute and to get an idea of the atomizer gas setting needed to print a certain amount of ink (see Fig. 6). For measuring the mass flow a small piece of aluminum foil was weighted then put under the aerosol beam for five minutes and then weighted again, the difference showed the amount of varnish coming through the nozzle on a respective atomizer setting.

The first thought, that the higher the atomizer gas setting was, the higher the mass flow could be rebutted. For every ink used, there is a specific window of maximum throughput. Exceeding this maximum leads to the building of droplets on the front of the nozzle, which continuously grows and subsequently suspends the process (see Fig. 7 left hand side). The highest throughputs could be reached by using gas settings between 900 and $1200 \mathrm{sccm}$.

$\mathrm{J}+\mathrm{S}$ and EpoCore showed very similar results and the process itself was very stable without building droplets on the tip of the nozzle when the atomizer gas did not exceed $1400 \mathrm{sccm}$ respectively $1200 \mathrm{sccm}$. Using OrmoCore the highest throughput could be generated, but over $800 \mathrm{sccm}$ massive droplet building started leading to a dripping out of the nozzle. Loctite Ablelux OGR-150THTG had the lowest mass flow, which was too low to do further printing tests.

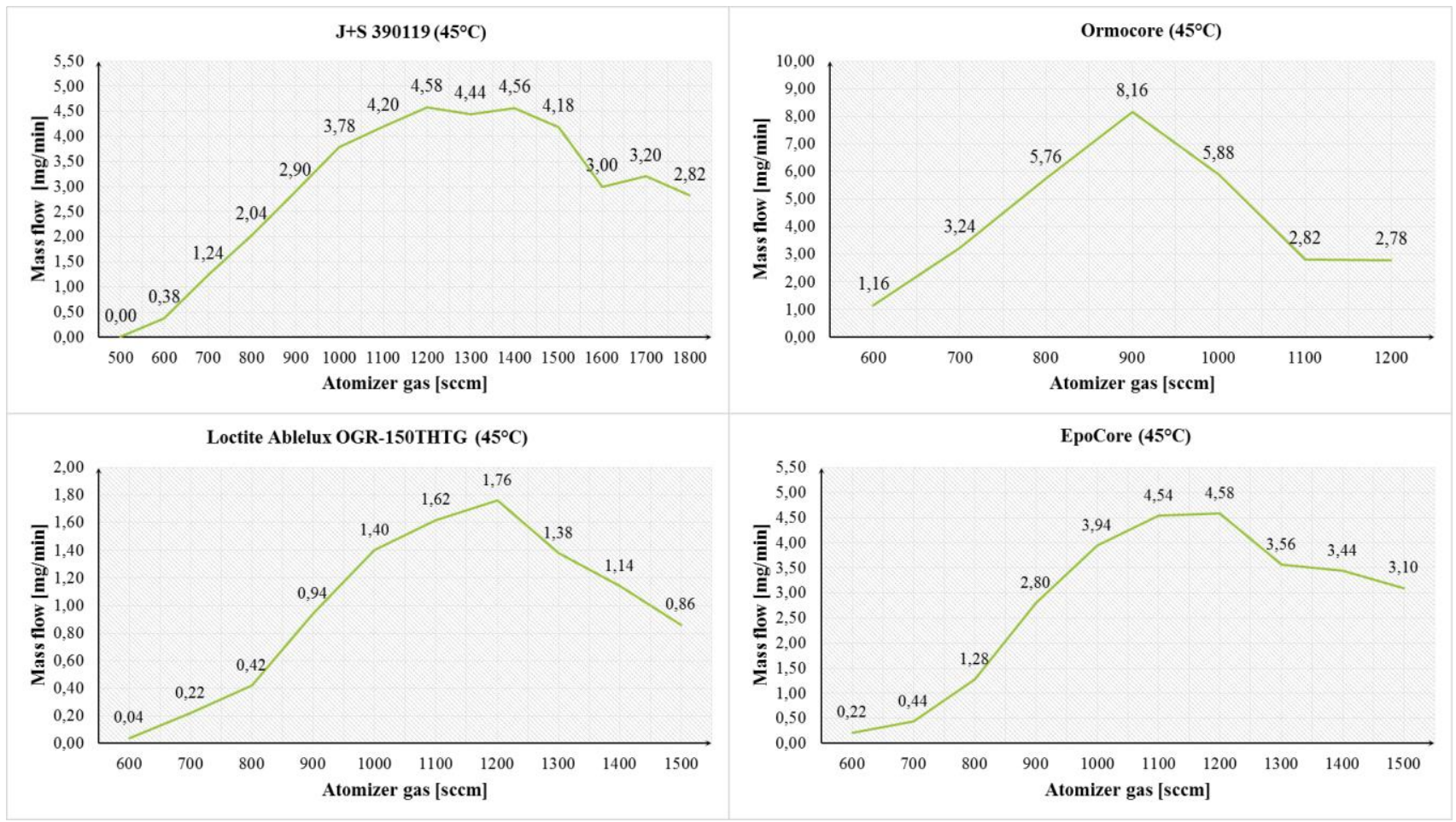

Figure 6. Mass flow measurement results of J+S 390119, Ormocore, Loctite Ablelux OGR-150THTG and EpoCore, preheated on $45^{\circ} \mathrm{C}$, for different atomizer gas settings.

To know the throughput at particular atomizer gas setting is also important for printing the maximum amount of varnish between the conditioning lines without flooding them (see Fig. 7 right hand side). The contact angle increases to a maximum of about $60^{\circ}$, after that the surface energy of the conditioning lines is not low enough to keep the liquid varnish between them. 

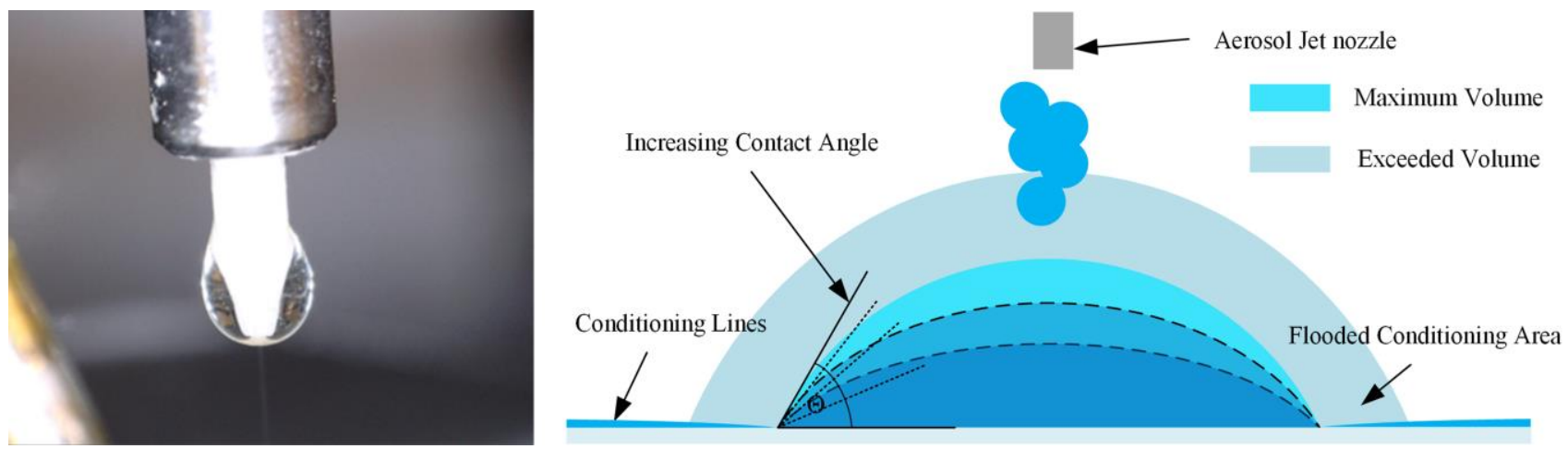

Figure 7. Left: Droplet building on the tip of the nozzle; Right: Increasing cross section of a printed polymer waveguide forced by conditioning the substrate material

\section{RESULTS AND CHALLENGES}

\subsection{Geometrical and optical characterization}

To define the printed structures geometrically a Keyence laser-scanning microscope VK 9710 was used. With this, it was possible to measure the cross section and the surface roughness of the printed patterns (see Fig. 8). The length of the investigated waveguide was $60 \mathrm{~mm}$ with $66 \mu \mathrm{m}$ in height and $328 \mu \mathrm{m}$ in width. Therefore the aspect ratio was 0.2 more than half the possible value. With a surface roughness of $0.06 \mu \mathrm{m}$, the waveguide has a very smooth surface, which is important for transferring light with the lowest optical loss possible. The attenuation and so the propagation loss was also measured, using a $10 \mu \mathrm{m}$ launching fiber (NA $=0.1$ ) and a $400 \mu \mathrm{m}$ detection fiber $(\mathrm{NA}=0.39$ ), the result was $0.7 \mathrm{~dB} / \mathrm{cm}$.
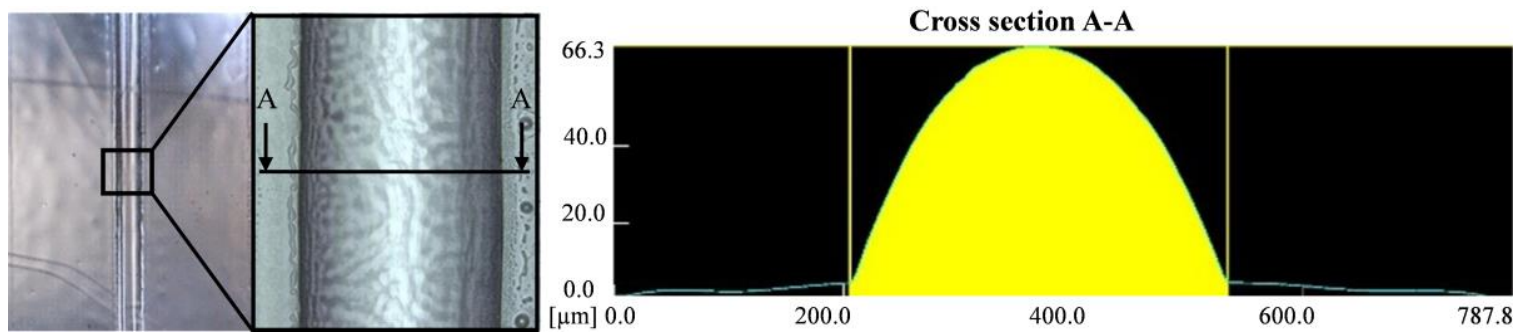

Figure 8. From left to right, the same printed POW: Real image; Laser scanning image top view; Laser scanning image cross section

\subsection{Future challenges}

There are few challenges, which have to be faced in the future:

1) The repeatability of the Flexographic printing has to be improved. The barrier lines need to have straight edges over the complete printing distance to avoid droplet building in the next process step. Further investigations have to be performed to know the difference between the theoretical and the realistic distance between the printed conditioning lines.

2) The repeatability of the Aerosol Jet Printing has to be improved. On the one hand, the complete machine setup has to be protected from UV-light, to prevent the used varnishes from curing inside the printer. On the other hand, the investigated machine parameters, methods for substrate handling and curing strategies, have to be standardized.

3) A suitable cladding material has to be found which matches the surface and refractivity requirements of the clear varnish (J+S 390119), with which so far the best results could be achieved.

4) Further both of the materials, the conditioning lines and the core/cladding combination, have to be tested. 


\section{CONCLUSION}

This paper shows the manufacturing of polymer optical waveguides by using the combination of Flexographic and Aerosol Jet Printing. Different ways of surface treatment were tested based on past studies in combination with printability of the varnish materials. After that, the machine settings were presented and different mass flow measurements were performed and detailed. With this background, it is possible to build up POWs with an attenuation of $0.7 \mathrm{~dB} / \mathrm{cm}$, a smooth parabolic surface and straight edges. Further investigations will be done to improve the attenuation and to obtain better understanding on how the machine settings affect this parameter. Combining this information and setting up a standardized repeatable process has to be the next step.

\section{AKNOWLEDGEMENT}

The authors would like to thank the "Deutsche Forschungsgemeinschaft (DFG)" for funding the research group and therefore providing the opportunity of doing fundamental progress in this seminal field of technology. The authors are wholly responsible for this publication.

\section{REFERENCES}

[1] "2015 IPC International Technology Roadmap for Electronic Interconnections," Part D - Interconnections and Substrates, Section 6 - Electrical and Optical Performance, 2015.

[2] Kim, E., Whitesides, G. M., Lee, L. K., Smith, S. P., Prentiss, M., "Fabrication of Arrays of Channel Waveguides by Self-Assembly Using Patterned Organic Monolayers as Templates,” Adv. Mater. 1996, 45(3), 123-13 (2000).

[3] Wolfer, T., Bollgruen, P., Mager, D., Overmeyer, L., Korvink, J. G., "Printing and preparation of integrated optical waveguides for optronic sensor networks," Mechatronics, (2015).

[4] Hörber, J., Goth, C., Franke, J., Hedges, M., "Electrical Functionalization of Thermoplastic Materials by Aerosol Jet Printing," Proceedings of the 13th Electronics Packaging Technology Conference (EPTC), Singapore, pp. 813-818 (2011).

[5] Reitberger, T., Hoerber, J., Schramm, R., Sennefelder, S., Franke, J., "Aerosol Jet ${ }^{\circledR}$ Printing of Optical Waveguides," Proceedings of the 38th International Spring Seminar on Electronics Technology (ISSE), Eger, Hungary; (2015).

[6] King, B.H., et al, "Characterizing Aerosol Jet ${ }^{\circledR}$ Multi-Nozzle Process Parameters for Non-Contact Front Side Metallization of Silicon Solar Cells," Photovoltaic Specialists Conference (PVSC), 34th IEEE, (2009).

[7] Verheecke, W., Van Dyck, M., Vogeler, F., Voet, A., Valkenaers, H., "Optimizing Aerosol Jet@ Printing of Silver Interconnects on Polyimide Film for Embedded Electronics Applications," 8th International DAAAM Baltic Conference "INDUSTRIAL ENGINEERING", Tallinn, Estonia, (2012). 